\title{
Effectiveness of Retropubic Tension-Free Vaginal Tape and Transobturator Inside-Out Tape Procedures in Women With Overactive Bladder and Stress Urinary Incontinence
}

\author{
Ji-Yeon Han, Myung-Soo Choo ${ }^{1}$, Young-Suk Lee ${ }^{2}$, Ju Tae Seo ${ }^{3}$, Jang Hwan Kim², Young Ho Kim ${ }^{5}$ Kyu-Sung Lee ${ }^{6}$ \\ Department of Urology, Pusan National University Yangsan Hospital, Yangsan; \\ ${ }^{1}$ Department of Urology, Asan Medical Center, University of Ulsan College of Medicine, Seoul; \\ ${ }^{2}$ Department of Urology, Samsung Changwon Hospital, Sungkyunkwan University School of Medicine, Changwon; \\ ${ }^{3}$ Department of Urology, Cheil General Hospital \& Women's Healthcare Center, Kwandong University College of Medicine, Seoul; \\ ${ }^{4}$ Department of Urology, Yonsei University College of Medicine, Seoul; \\ ${ }^{5}$ Department of Urology, Soonchunhyang University Bucheon Hospital, Bucheon; \\ ${ }^{6}$ Department of Urology, Samsung Medical Center, Sungkyunkwan University School of Medicine, Seoul, Korea
}

\begin{abstract}
Purpose: We compared the effectiveness of the retropubic tension-free vaginal tape (TVT) and the transobturator inside-out tape (TVT-O) in treating symptoms of overactive bladder (OAB) in women with stress urinary incontinence (SUI).

Methods: Women with urodynamic SUI and OAB (mean urgency episodes $\geq 1$ and frequency $\geq 8 / 24$ hours on a 3-day voiding diary) were assigned to the TVT or TVT-O group. Preoperative measures were based on a urodynamic study, 3-day voiding diary, the Bristol Female Lower Urinary Tract Symptoms questionnaire (BFLUTS $\mathrm{SF}_{\mathrm{F}}$ ), and the urgency perception scale (UPS). At 12 postoperative months, the 3 -day voiding diary, symptoms questionnaire, patient satisfaction, and standing stress test were assessed. The primary endpoint was change in the number of urgency episodes/24 hours from baseline to 12 months.

Results: In this group of 132 women, 42 received TVT and 90 received TVT-O. The mean urgency episodes/ 24 hours decreased from $6.3 \pm 5.5$ to $1.6 \pm 3.2$ in the TVT group and from $5.1 \pm 4.4$ to $1.8 \pm 3.0$ in the TVT-O group. The mean percent change was significantly greater after TVT than after TVT-O $(73 \%$ vs. $60 \%, \mathrm{P}=0.049)$. All subscales of BFLUTS sF and UPS were significantly improved using either method, with significantly greater improvement seen in the quality of life (QoL) domain after TVT $(\mathrm{P}=$ 0.002). There were no significant differences in the cure and satisfaction rates between the two groups.

Conclusions: Intervention with the TVT or the TVT-O significantly improved symptoms of OAB in women with SUI and OAB. Urgency and QoL significantly improved after TVT compared with that after TVT-O.
\end{abstract}

Keywords: Overactive urinary bladder; Stress urinary incontinence

\section{INTRODUCTION}

Since its introduction as a minimally invasive procedure, the midurethral sling (MUS) has become the most widely used treatment for female stress urinary incontinence (SUI). After more than 10 years' follow-up, retropubic tension-free vaginal tape (TVT) has shown to yield long-term cure rates of $84-90 \%$ $[1,2]$, and transobturator tape (TOT) procedures have compa- rable effectiveness to that of TVT $[3,4]$. Overactive bladder (OAB) symptoms can also be cured during operation for incontinence, although no satisfactory explanation can be provided for this. Approximately $50 \%$ of women suffering from SUI have coexisting symptoms of OAB [5,6]. In addition, those who undergo surgery for SUI have high expectations of a good outcome; in particular, patients expect that their OAB symptoms should be resolved as well [7]. If these symptoms are not
Corresponding author: Kyu-Sung Lee

Department of Urology, Samsung Medical Center, Sungkyunkwan University School of Medicine, 81 Irwon-ro, Gangnam-gu, Seoul 135-710, Korea Tel: +82-2-3410-3554 / Fax: +82-2-3410-3027 / E-mail: ksleedr@skku.edu Submitted: September 24, 2013 / Accepted after revision: September 26, 2013
This is an Open Access article distributed under the terms of the Creative Commons Attribution Non-Commercial License (http://creativecommons.org/licenses/by-nc/3.0/) which permits unrestricted non-commercial use, distribution, and reproduction in any medium, provided the original work is properly cited. 
resolved, patients may consider the surgery to be ineffective. Moreover, the coexistence of OAB and SUI reduces patient quality of life (QoL) more than that experienced by patients with SUI alone $[8,9]$. For these reasons, it is important to assess the effect of surgery for SUI on the symptoms of OAB.

The cure rate of preoperative urgency or urgency urinary incontinence (UUI) symptoms following MUS varies from 23\% to $75 \%$ [10-14]. Most studies on changes in OAB after MUS focused on women who underwent surgery primarily for SUI. In these studies, symptoms of $\mathrm{OAB}$ were not the primary outcome measures, and standardized, validated tools for assessing $\mathrm{OAB}$ were not used. Therefore, the objective of the present study was to compare the effectiveness of the TVT and transobturator vaginal tape inside-out (TVT-O) on OAB symptoms in women with both SUI and OAB.

\section{MATERIALS AND METHODS}

\section{Design}

This was a prospective, multicenter, comparative study performed at 10 institutions. The subjects received detailed information about the study procedure and provided written consent to participate.

\section{Population}

Women at 18 years of age and older with both urodynamic SUI and $\mathrm{OAB}$ were eligible for inclusion in the study. $\mathrm{OAB}$ was defined as urgency ( $\geq 3$ points on a five-point urinary sensation scale [USS]; 1 , no urgency to 5 , urgency incontinence) [15] more than one time per day and more than eight times per day on a 3-day voiding diary. Patients were excluded if they had a neurological disease or pelvic organ prolapse stage $\geq 2$, or if they were being treated with any behavioral therapy, anticholinergic drug for $\mathrm{OAB}$, or any drug treatment that might affect micturition 14 days prior to enrollment and throughout the trial.

\section{Procedure}

Consenting patients were assigned to the TVT or TVT-O for $\mathrm{OAB}$ symptoms based on the surgeon's preference at a given institution. The TVT and TVT-O procedures were performed by experienced surgeons using standard techniques provided by Ulmsten et al. [16] and de Leval [17]. A combination of light sedation and local anesthesia was normally used, but general or spinal anesthesia was also used according to each center's protocols.

\section{Assessment}

The preoperative protocol included clinical, neurological, and urogynecological examinations, a 3-day voiding diary, including an urgency scale, and a urodynamic study. The urodynamic study was performed in accordance with International Continence Society standards [18]. Scored forms of the Bristol Female Lower Urinary Tract Symptoms questionnaire $\left(\right.$ BFLUTS $_{\mathrm{SF}}$ ) [19] and Urgency Perception Scale (UPS) [20] were analyzed. At 12 months after the operations, outcome measures included a 3 -day voiding diary recording the urgency scale, symptom questionnaires, standing stress test, and patient's satisfaction assessment by a data collector. The objective cure of SUI was defined as a negative standing stress test, while the subjective cure was based on responses on the Sandvik questionnaire [21]. Cure of UUI and urgency were defined as no UUI and no urgency based on the voiding diary. Twelve months after the surgery, patients were asked to describe their overall satisfaction using answers such as very satisfied, satisfied, or not satisfied. All patients, except those self-described as not satisfied, were considered satisfied.

The primary endpoint was the change in number of urgency episodes per 24 hour from the baseline to the 12-month postoperative visit. The secondary endpoints were the objective cure of SUI, patient satisfaction with treatment, the changes in symptom questionnaire answers (BFLUTS $S_{\mathrm{SF}}$ and UPS), and voiding function changes from baseline to the 12 -month postoperative visit.

\section{Statistical Analysis}

SPSS ver. 12.0 (SPSS Inc., Chicago, IL, USA) was used for the data management and analysis. The Wilcoxon, Fisher exact, and chi-square tests were used to test homogeneity between the TVT and TVT-O groups. Within-group comparisons were analyzed using the Wilcoxon test or a paired t-test as appropriate. A P-value $<0.05$ indicated significance.

\section{RESULTS}

We assessed 158 consecutive patients in this study. Among these, 132 patients (42 in the TVT group and 90 in the TVT-O group) were included in the efficacy analysis (Fig. 1). The demographic and preoperative characteristics were similar between groups (Table 1).

After the operation, the mean number of urgency episodes per 24 hours decreased from $6.3 \pm 5.5$ to $1.6 \pm 3.2$ in the TVT 


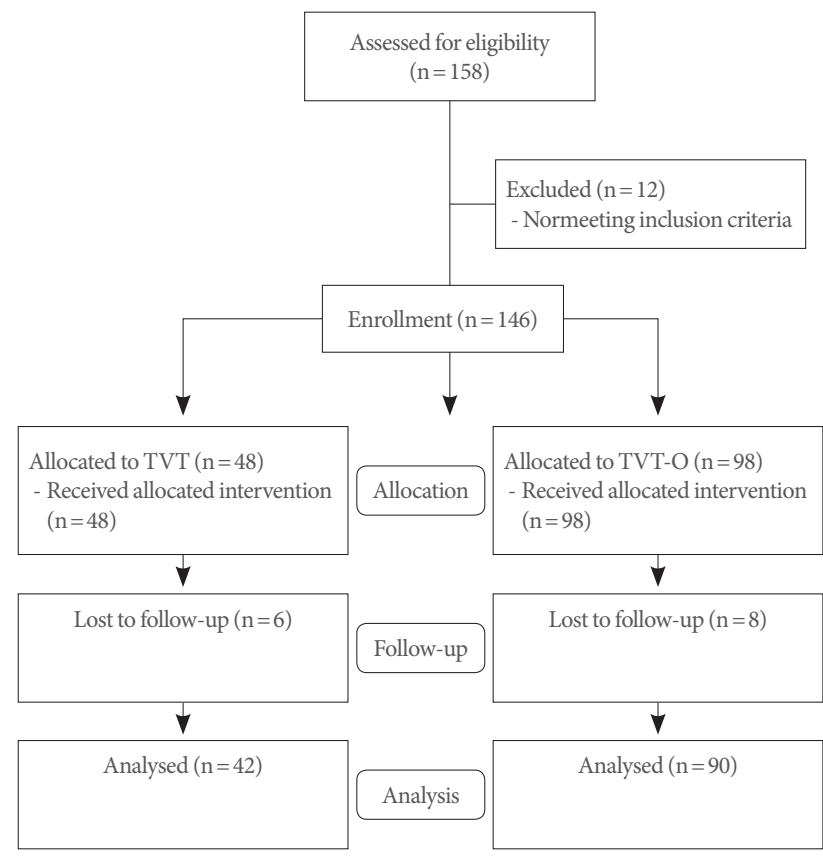

Fig. 1. Participation flowchart. TVT, tension-free vaginal tape; TVT-O, transobturator inside-out tape.

group and from $5.1 \pm 4.4$ to $1.8 \pm 3.0$ in the TVT-O group. The mean changes in urgency were greater in the TVT group than in the TVT-O group ( $-4.7 / 24$ hours vs. $-3.3 / 24$ hours), but the difference was not significant $(\mathrm{P}=0.216)$. When mean percent changes in voiding diary parameters were compared, only the mean percent change in the number of urgency episodes was found to differ between groups, and was significantly greater in the TVT than in the TVT-O group ( $74 \%$ vs. $60 \%, \mathrm{P}=0.049)$ at 12 months (Fig. 2). The overall objective cure rates for SUI were 95.2\% (40/42) in the TVT and 92.2\% (83/90) in the TVT-O group $(\mathrm{P}=0.718)$. Subjective cure rates for SUI were $85 \%(34 / 40)$ in the TVT and 79.6\% (70/88) in the TVT-O group $(\mathrm{P}=0.340)$. All subscales of BFLUTS $S_{\mathrm{SF}}$ (voiding, filling, incontinence, sexual function, and QoL) were improved in both groups, but the improvement in the QoL domain was significantly higher in the TVT group ( $\mathrm{P}=0.002)$ (Fig. 3).

Table 2 summarizes changes in the voiding diary and uroflowmetry parameters and between the TVT and TVT-O groups. In both groups, urination frequency and nocturia as well as urgency and UUI were significantly improved after surgery. The severity of the urgency and the maximum voided volume were significantly decreased after surgery, while the changes in the TVT group were significantly greater than those in the TVT-O group $(\mathrm{P}=0.049$ and $\mathrm{P}=0.002$, respectively).

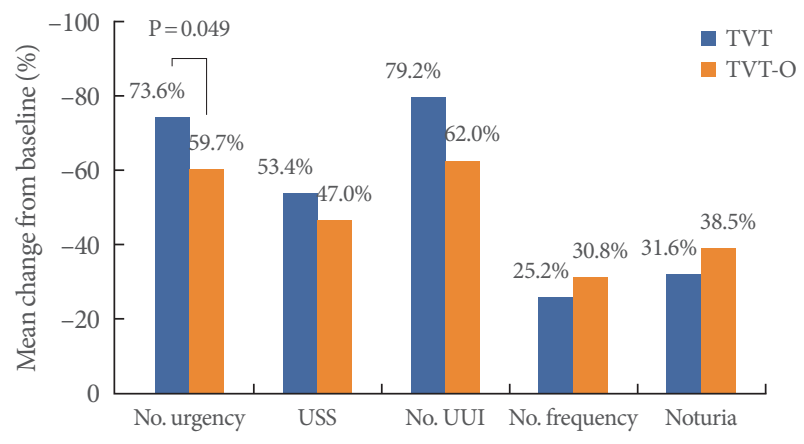

Fig. 2. Mean percent changes in voiding diary parameters from baseline to 12 months. TVT, tension-free vaginal tape; TVT-O, transobturator inside-out tape; USS, urinary sensation scale; UUI, urgency urinary incontinence.

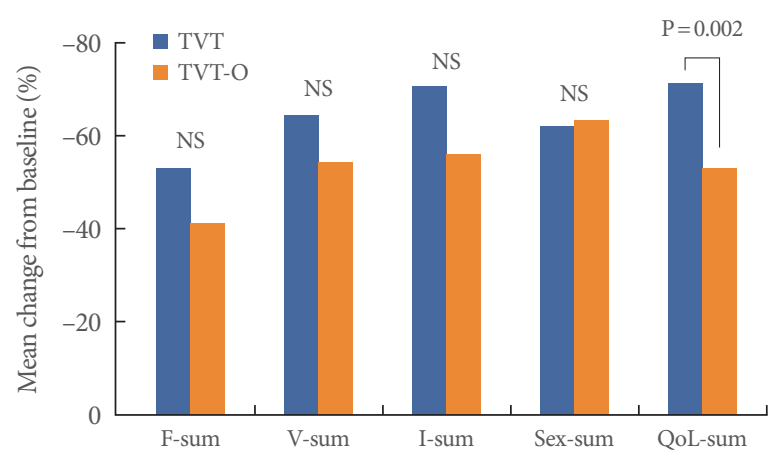

Fig. 3. Mean percent change in Bristol Female Lower Urinary Tract Symptoms questionnaire from baseline to 12 months. F, filling; V, voiding; I, incontinence; Sex, sex function; QoL, quality of life; NS, not significant; TVT, tension-free vaginal tape; TVT-O, transobturator inside-out tape.

The cure rates for urgency (53\% for TVT [20/38] vs. $51 \%$ for TVT-O [43/84], $\mathrm{P}=0.809$ ) and those for UUI (55\% for TVT [21/38] vs. $52 \%$ for TVT-O [44/84], $\mathrm{P}=0.836$ ) did not differ significantly between the two groups. After surgery, UPS was also significantly improved, and the improvement rates did not differ between the groups. The rates of patient satisfaction were $95.2 \%$ in the TVT group (40/42) and $96.7 \%(87 / 90)$ in the TVT-O group $(\mathrm{P}=0.653)$.

\section{DISCUSSION}

In this study, we prospectively compared the effectiveness of TVT and TVT-O in treating OAB symptoms using validated objective and subjective measures in women with both SUI and OAB. Both TVT and TVT-O resulted in significant improvement in $\mathrm{OAB}$ symptoms. However, the number of urgency epi- 
Table 1. Baseline characteristics of the intention-to-treat group $(n=132)$

\begin{tabular}{|c|c|c|c|}
\hline Characteristic & $\operatorname{TVT}(n=42)$ & TVT-O $(\mathrm{n}=90)$ & P-value \\
\hline Age (yr) & $52.5 \pm 8.8(35.0-72.0)$ & $52.3 \pm 10.0(35.0-80.0)$ & $0.710^{\mathrm{a})}$ \\
\hline $\operatorname{BMI}\left(\mathrm{kg} / \mathrm{m}^{2}\right)$ & $24.6 \pm 2.9(17.0-34.4)$ & $24.4 \pm 2.6(17.4-34.6)$ & $0.744^{\mathrm{a})}$ \\
\hline Parity & $2.3 \pm 1.0(0-5)$ & $2.6 \pm 1.1(0-5)$ & $0.380^{\mathrm{a})}$ \\
\hline \multicolumn{4}{|l|}{ Hormonal status } \\
\hline Menopausal & $20(47.6)$ & $42(47.2)$ & $0.924^{\mathrm{b})}$ \\
\hline On ERT & $3(15)$ & $6(16)$ & $0.628^{\mathrm{b})}$ \\
\hline \multicolumn{4}{|l|}{ Voiding diary } \\
\hline Frequency/24 hr & $10.8 \pm 1.8(8.0-14.7)$ & $11.4 \pm 3.0(8.0-24.7)$ & $1.000^{\mathrm{a})}$ \\
\hline Nocturia/24 hr & $2.0 \pm 1.2(0-4.0)$ & $1.9 \pm 1.5(0-7.7)$ & $0.614^{\mathrm{a})}$ \\
\hline Urgency/24hr & $6.3 \pm 5.5(1-23.3)$ & $5.1 \pm 4.4(1-23.0)$ & $0.388^{\mathrm{a})}$ \\
\hline UUI & $30(71.4)$ & $56(62.2)$ & $0.301^{\mathrm{b})}$ \\
\hline Severity of urgency/voiding & $1.7 \pm 1.4(0.1-4.3)$ & $1.3 \pm 1.3(0.1-4.9)$ & $0.145^{\mathrm{a})}$ \\
\hline Maximal voided volume & $316.4 \pm 125.2(150.0-800.0)$ & $296.9 \pm 84.8(140.0-600.0)$ & $0.745^{\mathrm{a})}$ \\
\hline \multicolumn{4}{|l|}{ Urodynamic parameters } \\
\hline Peak urinary flow (mL/sec) & $24.1 \pm 10.1(9.5-52.3)$ & $24.3 \pm 13.3(6.7-52.9)$ & $0.841^{\text {a) }}$ \\
\hline Voided volume (mL) & $230.9 \pm 84.0(126.0-398.1)$ & $242.7 \pm 133.1(122.0-675)$ & $0.986^{\mathrm{a}}$ \\
\hline Post-voided residual (mL) & $10.5 \pm 9.4(0-43.0)$ & $19.86 \pm 29.7(0-171.0)$ & $0.839^{\mathrm{a})}$ \\
\hline $\operatorname{ALPP}\left(\mathrm{cmH}_{2} \mathrm{O}\right)$ & $93 \pm 36(36-181)$ & $89 \pm 93(21-209)$ & $0.833^{\mathrm{a})}$ \\
\hline DO & $6 / 26(23)$ & $15 / 82(18)$ & $0.380^{c)}$ \\
\hline
\end{tabular}

Values are presented as mean \pm standard deviation (range) or number (\%).

TVT, tension-free vaginal tape; TVT-O, transobturator inside-out tape; BMI, body mass index; ERT, estrogen replacement therapy; UUI, urinary urgency incontinence; ALPP, abdominal leak point pressure; DO, detrusor overactivity.

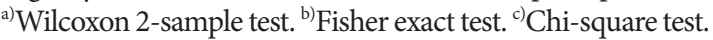

sodes, urgency grade, and maximum voided volume greatly improved with TVT than with TVT-O. Furthermore, TVT was associated with higher improvement postoperative QoL compared with TVT-O. We assume that the greater effectiveness of TVT as compared to TVT-O in treating OAB produced a correspondingly greater improvement in QoL.

To date, the relative effectiveness of the route of MUS procedures for OAB in SUI patients remains controversial. Therefore, the cure rate of $\mathrm{OAB}$ symptoms varies wide depending on the different parameters, unlike the parameters considered for SUI symptoms. Botros et al. [10] found that for UUI, the TOT produces a higher success rate than TVT or the suprapubic arc sling procedure. In addition, $14-16 \%$ of patients with preoperative UUI who underwent TVT or SPARC experienced worsening of their symptoms compared to only $6 \%$ of those treated by TOT. Porena et al. [22] reported that symptoms of storage, including daytime frequency, nocturia, and urgency, significantly improved or disappeared following treatment with TOT; however, these symptoms persisted in $24 \%$ of patients compared to $44 \%$ in patients treated with TVT. However, in another comparative study of TVT and TVT-O for lower urinary tract symptoms, changes in voiding and storage symptom scores on the American Urological Association Symptom Index before and after the procedure did not differ significantly [23]. In a recent meta-analysis on the effectiveness of MUS for mixed urinary incontinence, no significant difference in UUI cure was observed between the tapes used by the retropubic or transobturator routes [24].

It is unclear why the effects on $\mathrm{OAB}$ symptoms, including UUI, differ when retropubic and transobturator routes of midurethral tape replacement are used. One explanation may be because of the differences in the angle between the two limbs of the mesh used in TVT and TVT-O. SUI can induce and/or increase detrusor instability when intra-abdominal pressure is increased. Urine then enters the proximal urethra, increasing bladder activity and stimulating the urethral afferents [25]. An ultrasonographic study showed that, at rest, the tape is more 
Table 2. Changes in voiding diary and uroflowmetry parameters following TVT and TVT-O

\begin{tabular}{|c|c|c|c|c|c|}
\hline \multirow{2}{*}{ Variable } & \multicolumn{2}{|c|}{ TVT } & \multicolumn{2}{|c|}{ TVT-O } & \multirow{2}{*}{ P-value } \\
\hline & Preop & Postop & Preop & Postop & \\
\hline Urgency/24 hr & $6.3 \pm 5.5$ & $1.6 \pm 3.2^{\mathrm{b})}$ & $5.1 \pm 4.4$ & $1.8 \pm 3.0^{\mathrm{b})}$ & 0.216 \\
\hline \multicolumn{6}{|l|}{ Voiding diary } \\
\hline $\mathrm{UUI} / 24 \mathrm{hr}$ & $3.2 \pm 2.7$ & $0.6 \pm 1.0^{\mathrm{b})}$ & $2.7 \pm 2.4$ & $1.0 \pm 1.6^{\mathrm{b})}$ & 0.096 \\
\hline Micturition/24 hr & $10.8 \pm 1.8$ & $7.9 \pm 2.4^{\mathrm{b})}$ & $11.4 \pm 3.0$ & $7.7 \pm 2.9^{b)}$ & 0.407 \\
\hline Nocturia & $2.0 \pm 1.2$ & $0.9 \pm 1.0^{\mathrm{b})}$ & $1.9 \pm 1.5$ & $1.0 \pm 1.0^{\mathrm{b})}$ & 1.000 \\
\hline Urgency severity & $1.7 \pm 1.4$ & $1.0 \pm 1.3^{\mathrm{b})}$ & $1.3 \pm 1.3$ & $0.9 \pm 1.3^{b)}$ & 0.049 \\
\hline Maximal voided volume (mL) & $316.4 \pm 125.2$ & $382.4 \pm 152.2^{b)}$ & $296.9 \pm 84.8$ & $311.2 \pm 88.3$ & 0.041 \\
\hline \multicolumn{6}{|l|}{ Uroflowmetry } \\
\hline Voided volume (mL) & $230.9 \pm 84.0$ & $238.7 \pm 145.0$ & $242.7 \pm 133.1$ & $274.1 \pm 100.1^{\mathrm{b})}$ & 0.193 \\
\hline $\mathrm{Q}_{\max }(\mathrm{mL} / \mathrm{sec})$ & $24.1 \pm 18.7$ & $18.7 \pm 8.2^{\mathrm{b})}$ & $24.4 \pm 10.3$ & $22.9 \pm 9.3$ & 0.077 \\
\hline $\operatorname{PVR}(\mathrm{mL})$ & $10.5 \pm 9.4$ & $24.5 \pm 37.3$ & $19.9 \pm 29.7$ & $21.2 \pm 21.1$ & 0.673 \\
\hline
\end{tabular}

Values are presented as mean \pm standard deviation (range).

TVT, tension-free vaginal tape; TVT-O, transobturator inside-out tape; UUI, urinary urgency incontinence; $\mathrm{Q}_{\max }$, maximum flow rate; PVR, postvoid residual.

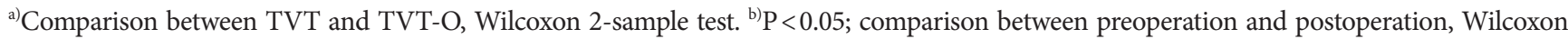
2-sample test.

open in the TOT and TVT-O treatments than in the TVT, and that a larger angle at rest is significantly associated with recurrent SUI [26]. At maximum retention, the angle closes through the traction of the two arms, with the lavatory plate raising the anterior vaginal wall. As intra-abdominal pressure increases, these tapes work as a dynamic closing mechanism that affects the urethra. Therefore, we hypothesize that the greater angular closure provided by the TVT may more effectively prevent urine from entering the upper posterior urethra, thereby improving reflex urgency to a greater degree than TVT-O. To support our hypothesis, however, long-term data are needed to demonstrate a correlation between postoperative UUI and OAB symptoms and tape angulation.

The difference in the outcome of these procedures might alternatively depend on tape position. An appropriately performed TVT involves minimal dissection and placement of the tape tension-free around the midurethra. Rezapour and Ulmsten [14] assumed that because of this, the proximal part of the densely innervated urethra and bladder neck would be less compromised to the benefit of UUI. In the case of the TOT, however, a longer suburethral incision and a larger paraurethral dissection are needed compared to that needed in the TVT because the index finger has to enter the paraurethral dissection. A study conducted to verify whether the TOT tape did not move from the midurethral space, resulting in subsequent voiding difficul- ty [27] found no difference in tape position, even though the TOT was closer to the bladder neck than the TVT. The authors noted that their finding was not statistically significant, and that the study lacked the statistical power to detect a difference. Larger-scale studies are needed to establish the effect of tape position on changes in $\mathrm{OAB}$ symptoms via surgery.

The mixed symptoms observed may be due to a more severe form of incontinence caused predominantly by stress [28], explaining why mixed symptoms are resolved after successful MUS. When patients report a sudden loss of urine associated with urgency, this condition may represent SUI combined with waiting too long to void rather than true urgency or UUI. In addition, patients may adopt behaviors of frequency as a strategy to control SUI. Such patients can be cured after anti-incontinence surgery compared with those who have true severe UUI. Consistent with this theory, we found that frequency as well as urgency or UUI decreased significantly after surgery. However, this hypothesis does not easily explain why the TVT procedure would more readily resolve urgency symptoms. Despite the use of reliable tools to assess $\mathrm{OAB}$, including a validated questionnaire and UPS, we cannot exclude the possibility of data confounding by "pseudo-urge symptoms" [11].

Additional limitations of this study include its small population size, the relatively short follow-up period, and the fact that we allowed the surgeon to decide which procedure to perform 
by their respective preference for OAB symptoms instead of randomizing the patients. Thus, more surgeons preferred TVTO over TVT that may have introduced unknown variability into outcome measures. Although few meta-analyses have analyzed the effectiveness of MUS in mixed urinary incontinence, to date, the cure rate of $\mathrm{OAB}$ symptoms varies widely according to MUS procedures, and most patients have high expectations of being cured of their OAB symptoms. Therefore, patients were assigned to either the TVT or TVT-O group based on surgeon experience of treating OAB symptoms in patients with SUI, instead of randomizing the patients. In this study, however, the two groups were apparently well matched in baseline clinical characteristics.

In conclusion, the surgical insertion of TVT and TVT-O significantly improved the OAB symptoms in women with both SUI and OAB. In particular, urgency significantly decreased and QoL significantly improved following TVT compared with TVT-O. Based on these favorable outcomes, TVT may be reasonably proposed as a treatment option in counseling patients who have both SUI and OAB.

\section{CONFLICT OF INTEREST}

No potential conflicts of interest relevant to this article are reported.

\section{REFERENCES}

1. Nilsson CG, Palva K, Rezapour M, Falconer C. Eleven years prospective follow-up of the tension-free vaginal tape procedure for treatment of stress urinary incontinence. Int Urogynecol J Pelvic Floor Dysfunct 2008;19:1043-7.

2. Olsson I, Abrahamsson AK, Kroon UB. Long-term efficacy of the tension-free vaginal tape procedure for the treatment of urinary incontinence: a retrospective follow-up 11.5 years post-operatively. Int Urogynecol J 2010;21:679-83.

3. Angioli R, Plotti F, Muzii L, Montera R, Panici PB, Zullo MA. Tension-free vaginal tape versus transobturator suburethral tape: fiveyear follow-up results of a prospective, randomised trial. Eur Urol 2010;58:671-7.

4. Lee KS, Han DH, Choi YS, Yum SH, Song SH, Doo CK, et al. A prospective trial comparing tension-free vaginal tape and transobturator vaginal tape inside-out for the surgical treatment of female stress urinary incontinence: 1-year followup. J Urol 2007;177:214-8.

5. Teleman PM, Lidfeldt J, Nerbrand C, Samsioe G, Mattiasson A; WHILA study group. Overactive bladder: prevalence, risk factors and relation to stress incontinence in middle-aged women. BJOG 2004;111:600-4.

6. Wagg A, Das Gupta R, Assassa P, Shaw C, Mayne C, Martin M. Secondary-care treatment patterns in the UK for women with urinary incontinence. BJU Int 2005;96:839-42.

7. Mahajan ST, Elkadry EA, Kenton KS, Shott S, Brubaker L. Patientcentered surgical outcomes: the impact of goal achievement and urge incontinence on patient satisfaction one year after surgery. Am J Obstet Gynecol 2006;194:722-8.

8. Monz B, Chartier-Kastler E, Hampel C, Samsioe G, Hunskaar S, Espuna-Pons $\mathrm{M}$, et al. Patient characteristics associated with quality of life in European women seeking treatment for urinary incontinence: results from PURE. Eur Urol 2007;51:1073-81.

9. Davis TL, Lukacz ES, Luber KM, Nager CW. Determinants of patient satisfaction after the tension-free vaginal tape procedure. Am J Obstet Gynecol 2004;191:176-81.

10. Botros SM, Miller JJ, Goldberg RP, Gandhi S, Akl M, Beaumont JL, et al. Detrusor overactivity and urge urinary incontinence following trans obturator versus midurethral slings. Neurourol Urodyn 2007;26:42-5.

11. Choe JH, Choo MS, Lee KS. The impact of tension-free vaginal tape on overactive bladder symptoms in women with stress urinary incontinence: significance of detrusor overactivity. J Urol 2008;179: 214-9.

12. Han JY, Song C, Park J, Jung HC, Lee KS, Choo MS. A long-term study of the effects of the tension-free vaginal tape procedure for female stress urinary incontinence on voiding, storage, and patient satisfaction: a post-hoc analysis. Korean J Urol 2010;51:40-4.

13. Holmgren C, Nilsson S, Lanner L, Hellberg D. Long-term results with tension-free vaginal tape on mixed and stress urinary incontinence. Obstet Gynecol 2005;106:38-43.

14. Rezapour M, Ulmsten U. Tension-Free vaginal tape (TVT) in women with mixed urinary incontinence: a long-term follow-up. Int Urogynecol J Pelvic Floor Dysfunct 2001;12 Suppl 2:S15-8.

15. Coyne KS, Tubaro A, Brubaker L, Bavendam T. Development and validation of patient-reported outcomes measures for overactive bladder: a review of concepts. Urology 2006;68(2 Suppl):9-16.

16. Ulmsten U, Henriksson L, Johnson P, Varhos G. An ambulatory surgical procedure under local anesthesia for treatment of female urinary incontinence. Int Urogynecol J Pelvic Floor Dysfunct 1996; 7:81-5.

17. de Leval J. Novel surgical technique for the treatment of female stress urinary incontinence: transobturator vaginal tape inside-out. Eur Urol 2003;44:724-30.

18. Abrams P, Cardozo L, Fall M, Griffiths D, Rosier P, Ulmsten U, et 
al. The standardisation of terminology in lower urinary tract function: report from the standardisation sub-committee of the International Continence Society. Urology 2003;61:37-49.

19. Jackson S, Donovan J, Brookes S, Eckford S, Swithinbank L, Abrams P. The bristol female lower urinary tract symptoms questionnaire: development and psychometric testing. Br J Urol 1996;77:805-12.

20. Cardozo L, Coyne KS, Versi E. Validation of the urgency perception scale. BJU Int 2005;95:591-6.

21. Sandvik H, Hunskaar S, Seim A, Hermstad R, Vanvik A, Bratt H. Validation of a severity index in female urinary incontinence and its implementation in an epidemiological survey. J Epidemiol Community Health 1993;47:497-9.

22. Porena M, Costantini E, Frea B, Giannantoni A, Ranzoni S, Mearini $\mathrm{L}$, et al. Tension-free vaginal tape versus transobturator tape as surgery for stress urinary incontinence: results of a multicentre randomised trial. Eur Urol 2007;52:1481-90.

23. Ballert KN, Kanofsky JA, Nitti VW. Effect of tension-free vaginal tape and TVT-obturator on lower urinary tract symptoms other than stress urinary incontinence. Int Urogynecol J Pelvic Floor Dysfunct 2008;19:335-40.
24. Jain P, Jirschele K, Botros SM, Latthe PM. Effectiveness of midurethral slings in mixed urinary incontinence: a systematic review and meta-analysis. Int Urogynecol J 2011;22:923-32.

25. Jung SY, Fraser MO, Ozawa H, Yokoyama O, Yoshiyama M, De Groat WC, et al. Urethral afferent nerve activity affects the micturition reflex; implication for the relationship between stress incontinence and detrusor instability. J Urol 1999;162:204-12.

26. Chene G, Cotte B, Tardieu AS, Savary D, Mansoor A. Clinical and ultrasonographic correlations following three surgical anti-incontinence procedures (TOT, TVT and TVT-O). Int Urogynecol J Pelvic Floor Dysfunct 2008;19:1125-31.

27. de Tayrac R, Deffieux X, Resten A, Doumerc S, Jouffroy C, Fernandez H. A transvaginal ultrasound study comparing transobturator tape and tension-free vaginal tape after surgical treatment of female stress urinary incontinence. Int Urogynecol J Pelvic Floor Dysfunct 2006;17:466-71.

28. Bump RC, Norton PA, Zinner NR, Yalcin I; Duloxetine Urinary Incontinence Study Group. Mixed urinary incontinence symptoms: urodynamic findings, incontinence severity, and treatment response. Obstet Gynecol 2003;102:76-83. 